\title{
Prefrontal cognitive deficits in patients with schizophrenia treated with atypical or conventional antipsychotics
}

\author{
U. Müller ${ }^{\text {a,c, },}$, K. Werheid ${ }^{\text {b,d }}$, E. Hammerstein ${ }^{\text {a }}$, S. Jungmann ${ }^{\text {a }}$, T. Becker ${ }^{\text {a,e }}$ \\ ${ }^{a}$ Department of Psychiatry, University of Leipzig, Leipzig, Germany \\ ${ }^{\mathrm{b}}$ Department of Cognitive Neurology, University of Leipzig, Leipzig, Germany \\ ${ }^{\mathrm{c}}$ Departments of Psychiatry and Experimental Psychology, University of Cambridge, Downing Site, Cambridge CB2 $3 E B$, UK \\ ${ }^{\mathrm{d}}$ Department of Psychology, Humboldt University Berlin, Berlin, Germany \\ e Department of Psychiatry II, University of Ulm, Günzburg, Germany
}

Received 10 May 2004; accepted 24 August 2004

Available online 13 December 2004

\begin{abstract}
Forty-three patients with schizophrenia were investigated with a short neurocognitive screening battery focussing on working memory and executive functions. As compared to healthy controls, patients showed impairments in the modified card sorting test, in verbal fluency and all span tasks with exception of digit span forward. Patients who were treated with atypicals showed better performance in the digit ordering test (manipulation task) when compared to a group of patients who received conventional antipsychotics; this difference was not due to disease severity, age or education. Manipulation tasks might be useful for neurocognitive follow-up and intervention studies.

(C) 2004 Elsevier SAS. All rights reserved.
\end{abstract}

Keywords: Antipsychotic agents; Executive functions; Digit ordering test; Schizophrenia; Working memory

\section{Introduction}

Neurocognitive deficits are a core feature of schizophrenia and a good predictor of functional outcome $[1,4]$. Comprehensive neuropsychological testing of all relevant cognitive domains requires several hours and is not feasible in clinical practice. Short bedside batteries have been proposed that focus on working memory and executive functions [4]. These functions are preferentially mediated by frontostriatal networks and modulated by major neurotransmitters like dopamine [11,12].

Antipsychotic drugs are well-established to reduce psychotic symptoms via blockade of dopamine D2 receptors [6]. When compared to conventional neuroleptics the newer atypical drugs have fewer side effects and a more favourable impact on negative symptoms, cognitive deficits and social outcome of patients with schizophrenia $[4,9,13,14]$. However, there is still there is still some debate as to whether atypical antipsychotics are more effective in treating cognitive symptoms

\footnotetext{
* Corresponding author.

E-mail address: um207@cam.ac.uk (U. Müller).
}

when compared to lower doses of conventional neuroleptics [8]. And so far, it is also not clear which cognitive processes are influenced differentially by conventional and atypical antipsychotic medication.

The aim of this study was to evaluate a short prefrontal screening battery that detects relevant cognitive deficits in patients with schizophrenia and allows differentiating disease and treatment effects.

\section{Methods}

\subsection{Participants and design}

Forty-three patients (19 female; age $39.2 \pm 12.2$ years) with a diagnosis of schizophrenia according to ICD-10 diagnostic criteria for research [16] were recruited from consecutive admissions to the Department of Psychiatry, University Hospital Leipzig, and interviewed by two experienced psychiatrists (U.M., T.B.) to exclude major psychiatric or medical comorbidity and perform standardised psychopathological and diagnostic ratings. The mean duration of their disease 
was $10.3 \pm 10.7$ (range 0-38) years and the overall PANSS score was $62.5 \pm 14.6$ (range 40-94). They were tested at the end of or shortly after inpatient treatment on stable medication. Twenty-two patients $(51 \%)$ were treated with atypicals only (clozapine $[n=10$; mean dose 285 , range 150 $550 \mathrm{mg} /$ day], olanzapine $[n=8 ; 16.4,10-20 \mathrm{mg} /$ day], risperidone $[n=4 ; 4.3,1-6 \mathrm{mg} / \mathrm{day}]$, amisulpride $[n=3 ; 450$, $350-600 \mathrm{mg} / \mathrm{day}]$, or quetiapine $[n=2$; 550, 500$600 \mathrm{mg} /$ day], including combinations), whereas 21 patients (49\%) were treated with conventional neuroleptics (flupenthixol $[n=14$, mean 7.5 , range $2-17 \mathrm{mg} /$ day], haloperidol $[n=7 ; 10.2,4-21 \mathrm{mg} /$ day $]$, pimozide [ $n=1 ; 4 \mathrm{mg} /$ day $]$, or levomepromazine $[n=1,50 \mathrm{mg} /$ day], including combinations, e.g. medium dose flupenthixol or haloperidol plus clozapine or olanzapine). Treatment with anticholinergics or benzodiazepines was interrupted before cognitive testing, if possible. Psychiatric co-medication with antidepressants (no tricyclics) and hypnotics in the evening (antihistaminic drugs or benzodiazepines) was equally distributed between the two groups. At the time of testing, eight patients (seven on atypical vs. one on conventional antipsychotics) were employed, 12 (seven vs. five) were unemployed or in training and 23 ( 8 vs. 15 ) received a disability pension.

Twenty-seven healthy subjects from the volunteers' panel of the Max Planck Institute for Human Cognitive and Brain Sciences, Leipzig, served as controls and were matched for sex (12 female), age (38.2 \pm 13.9 , range $22-58$ years), and years of education.

Neurocognitive testing was performed in one session in a quiet wardroom. The protocol was approved by the Ethics Committee of the University of Leipzig and written informed consent was obtained from all participants.

\subsection{Neurocognitive testing}

A short neuropsychological testing battery of wellestablished paper and pencil tests was adapted from our previous studies in patients with Parkinson's disease and frontal lobe lesions $[11,15]$. A short description of all tasks is given in Table 1. Interestingly, our battery overlaps with the Brief
Assessment of Cognition in Schizophrenia (BACS) battery in four of seven tests [4]. To assess manipulation processes in working memory we were using the digit ordering span task that has been developed by our group: similar to digit spans forward and backward, number sequences of increasing length are presented and have to be recalled in ascending order [15].

\subsection{Data analysis and statistics}

Neuropsychological and demographical data were compared by means of ANOVA or nonparametric Mann-Whitney $U$-tests, depending on normal distribution of the data. Spearman's rank sums were used to calculate correlations.

\section{Results}

As compared to controls, patients with schizophrenia showed significant deficits in all tests with the exception of digit span forward (Table 1). Patients treated with atypical antipsychotics only showed significant better performance in the digit ordering test when compared to those on conventional neuroleptics $(5.0 \pm 0.9$ vs. $4.4 \pm 1.3 ; U=150.5$, $P<0.05)$; this difference is not due to age $(36.4 \pm 12.5$ vs. $42.2 \pm 11.3$ years) or disease severity as measured with the PANSS $(61.3 \pm 14.3$ vs. $63.8 \pm 15.1)$. There were no other significant differences between the two drug groups, with the exception of a trend for shorter disease duration (7.9 $\pm 10.7 \mathrm{vs}$. $12.8 \pm 10.3$ years) in the atypical group (Fig. 1).

In the patient group there were no significant correlations between cognitive and psychopathological parameters, with the exception of the PANSS disorganisation cluster. Higher disorganisation was correlated with lower performance on all manipulative spans (backward, ordering and reading) and categories in the modified card sorting test.

\section{Discussion}

We have shown that a short neurocognitive testing battery allows detecting deficits of working memory and executive

Table 1

Neurocognitive profile of patients with schizophrenia $(n=43)$ and healthy controls $(n=27)$ in the prefrontal battery (mean \pm S.D.)

\begin{tabular}{|c|c|c|c|c|c|}
\hline Task & Short description ( $[11,15]$ for details) & Patients & $\begin{array}{l}\text { Healthy } \\
\text { controls }\end{array}$ & $U$ & $P$ \\
\hline Digit span & Recall digit sequences of increasing length forward and backward & & & & \\
\hline -Forward & & $6.2 \pm 1.1$ & $6.5 \pm 1.1$ & 490.0 & $>0.10$ \\
\hline -Backward & & $4.0 \pm 1.0$ & $5.2 \pm 1.0$ & 208.5 & $<0.001$ \\
\hline Digit ordering span & Recall digit sequences in ascending order & $4.7 \pm 1.1$ & $6.4 \pm 0.8$ & 116.5 & $<0.001$ \\
\hline Reading span & Recall last words of short sentences & $2.5 \pm 1.2$ & $3.7 \pm 0.3$ & 150.0 & $<0.001$ \\
\hline Verbal fluency & Name words that begin with F, P, R or animals in $60 \mathrm{~s}$ & & & & \\
\hline -Literal & & $23.7 \pm 10.7$ & $30.0 \pm 10.8$ & 372.5 & $<0.02$ \\
\hline -Categorical & & $19.0 \pm 5.7$ & $26.2 \pm 8.1$ & 253.0 & $<0.001$ \\
\hline MCST & Sort cards according to changing rules (with error feedback) & & & & \\
\hline -Categories & & $4.8 \pm 2.0$ & $6.4 \pm 0.7$ & 281.0 & $<0.001$ \\
\hline -Perseverations & & $2.2 \pm 3.0$ & $0.4 \pm 0.8$ & 371.5 & $<0.01$ \\
\hline MWT-A & Cancel words that do not exist in German & $28.2 \pm 5.5$ & $31.8 \pm 2.2$ & 334.5 & $<0.01$ \\
\hline
\end{tabular}

MCST : Modified card sorting test; MWT-A : Mehrfachwortschatztest, version A (German NART equivalent). 


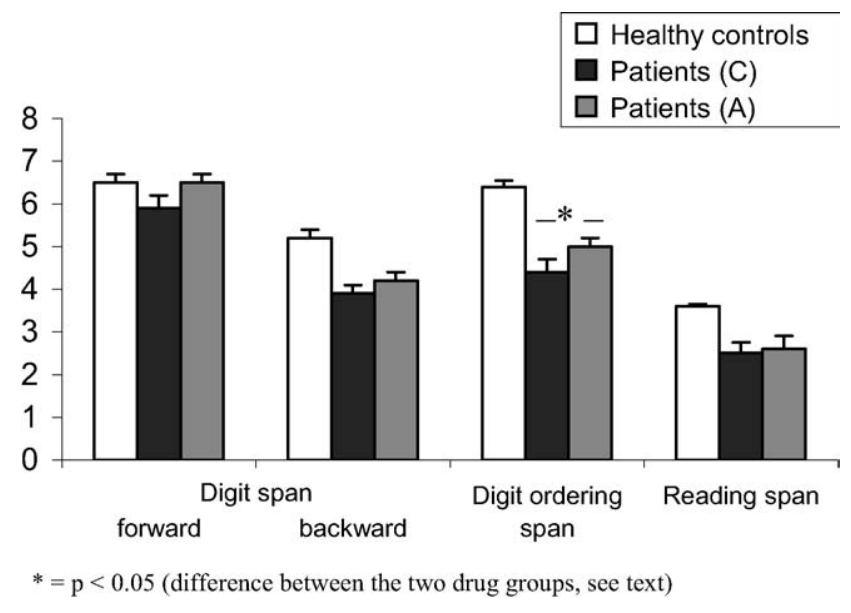

Fig. 1. Working memory (span task) performance of healthy controls $(n=27)$ and patients with schizophrenia treated with conventional $(\mathrm{C})(n=21)$ or atypical (A) $(n=22)$ antipsychotic drugs (mean \pm S.D.).

functions in patients with schizophrenia in a clinical routine setting. Most interestingly, patients treated with atypical antipsychotics performed better in the digit ordering test than patients treated with conventional antipsychotics.

Working memory performance correlates with other executive deficits and can be taken as an indicator of more general neurocognitive deficits in patients with schizophrenia $[4,10]$. Working memory involves not only the maintenance of information, but also a manipulative component, which may be more severely affected in schizophrenic patients [7]. In many everyday life situations (e.g. to participate in a discussion, to dial long telephone numbers, to operate machines or personal computers) it is necessary to manipulate bits of information in working memory. Correlations of performance in manipulation tasks and the PANSS disorganisation score reflect specific cognitive problems of patients with schizophrenia. Manipulation processes, as specifically captured by digit ordering, are improved by dopamine agonists [2], impaired by anticholinergic and glutamatergic drugs [2,5], correlate with degeneration of nigrostriatal neurons in Parkinson's disease [11] and may be a sensible parameter to detect neurotransmitter specific effects of cognitive enhancing treatment.

The following limitations of this study have to be considered: First, the cognitive results may be confounded by the use of several different antipsychotics in both the conventional and the atypical group. On the other hand the naturalistic design of our study reflects prescription habits in a typical European region. The increasing prescription of bettertolerated but more expensive atypicals in Germany and other European countries [3], will make it difficult to perform similar studies in the future. Second, the cross-sectional design of our study without follow-up investigations makes it difficult to distinguish an improvement of working memory processes by atypical medication from impairment by conventional drugs. Third, daily doses of haloperidol (and flupenthixol) used in our clinic were relatively high at the time of the study. A recent meta-analysis showed that lower doses
(2-5 mg/day) of haloperidol have beneficial effects on cognitive functioning when compared to placebo or no treatment in patients with schizophrenia [8]. It is unlikely that anticholinergic drugs or side effects confounded our findings, because none of the patients was treated with biperiden or amitriptyline at the time of neuropsychological testing. Fourth, there were more employed patients in the atypical group and more patients in the conventional group received a disability pension. It is not clear from these data, however, if better cognitive functioning favours employment or vice versa.

Although antipsychotic drugs have some benefit on cognitive function, further efforts to improve cognitive deficits in schizophrenia are required. There is a vital need for developing improved compounds for the treatment of cognitive deficits in schizophrenia. The combination of more specific cognitive enhancing drugs with cognitive remediation therapies might lead to better functional outcome in patients with schizophrenia [4].

This study contributes further evidence for a beneficial effect of atypical antipsychotics on prefrontal cognitive functions in schizophrenia. Further studies will investigate the predictive value of performance in the digit ordering and similar manipulation tasks for overall cognitive and social functioning.

\section{Acknowledgements}

This study was supported by the German Bundesministerium für Bildung und Forschung (BMBF)/Medical Faculty of the University of Leipzig (formel.1-13) and the Alexander von Humboldt-Foundation (Feodor Lynen-Fellowship awarded to U.M.).

\section{References}

[1] Addington J, Addington D. Neurocognitive and social functioning in schizophrenia: a 2.5 year follow-up study. Schizophr Res 2000;44: 47-56.

[2] Cooper JA, Sagar HJ, Doherty SM, Jordan N, Tidswell P, Sullivan EV. Different effects of dopaminergic and anticholinergic therapies on cognitive and motor function in Parkinson's disease: a follow-up study of untreated patients. Brain 1992;115:1701-25.

[3] Hamann J, Pfeiffer H, Leucht S, Kissling W. Are patients with schizophrenia under-treated with second-generation antipsychotics? A pilot study of the prescription practices of German psychiatrists. Pharmacopsychiatry 2003;36:309-12.

[4] Harvey PD, Green MF, Keefe RS, Velligan DI. Cognitive functioning in schizophrenia: a consensus statement on its role in the definition and evaluation of effective treatments for the illness. J Clin Psychiatry 2004;65:361-72.

[5] Honey RA, Turner DC, Honey GD, Sharar SR, Kumaran D, PomarolClotet E, et al. Subdissociative dose ketamine produces a deficit in manipulation but not maintenance of the contents of working memory. Neuropsychopharmacology 2003;28:2037-44.

[6] Kapur S. How antipsychotics become anti- 'psychotic'-from dopamine to salience to psychosis. Trends Pharmacol Sci 2004;25:402-6. 
[7] Kim J, Glahn DC, Nuechterlein KH, Cannon TD. Maintenance and manipulation of information in schizophrenia: further evidence for impairment in the central executive component of working memory. Schizophr Res 2004;68:173-87.

[8] Mishara AL, Goldberg TE. A meta-analysis and critical review of the effects of conventional neuroleptic treatment on cognition in schizophrenia: opening a closed book. Biol Psychiatry 2004;55: 1013-22.

[9] Möller HJ. Management of the negative symptoms of schizophrenia: new treatment options. CNS Drugs 2003;17:793-823.

[10] Moritz S, Andresen B, Jacobsen D, Mersmann K, Wilke U, Lambert M, et al. Neuropsychological correlates of schizophrenic syndromes in patients treated with atypical neuroleptics. Eur Psychiatry 2001;16:354-61.

[11] Müller U, Wächter T, Barthel H, Reuter M, Von Cramon DY. Striatal $\left[{ }^{123} \mathrm{I}\right] \beta$-CIT SPECT and prefrontal memory functions in Parkinson's disease. J Neural Transm 2000;107:303-19.
[12] Robbins TW. Chemical neuromodulation of frontal-executive functions in humans and other animals. Exp Brain Res 2000;133:130-8.

[13] Weickert TW, Goldberg TE, Marenco S, Bigelow LB, Egan MF, Weinberger DR. Comparison of cognitive performances during a placebo period and an atypical antipsychotic treatment period in schizophrenia: critical examination of confounds. Neuropsychopharmacology 2003;28:1491-500.

[14] Weiss EM, Bilder RM, Fleischhacker WW. The effects of secondgeneration antipsychotics on cognitive functioning and psychosocial outcome in schizophrenia. Psychopharmacology 2002;162:11-7.

[15] Werheid K, Hoppe C, Thöne A, Müller U, Müngersdorf M, Von Cramon DY. The adaptive digit ordering test: clinical application, reliability and validity of a verbal working memory test. Arch Clin Neuropsychol 2002;17:547-65.

[16] World Health Organisation (WHO). The ICD-10 classification of mental and behavioural disorders: diagnostic criteria for research. Geneva: WHO; 1992. 